\title{
Hiccups associated with switching from quetiapine to aripiprazole in an adolescent patient
}

\author{
Mert Besenek $^{1(0)}$ \\ 'Recep Tayyip Erdogan University, Rize Training and Research Hospital, Child and Adolescent Psychiatry Clinic, Rize - Turkey
}

\begin{abstract}
Aripiprazole has a distinct place amongst other antipsychotics with its partial agonist-antagonist effect on dopaminergic and serotonergic receptors. Several case reports are associating aripiprazole to hiccups, but most of them are in the adult age group. The exact pathophysiologic mechanisms underlying this side effect are still fairly unknown and case reports displaying this phenomenon in adolescent patients are scarce. We report an adolescent bipolar patient who developed hiccups right after panic-like symptoms while switching from quetiapine to aripiprazole. Switching from a more potent D2 and 5HT1A antagonist to aripiprazole seems to increase the risk of hiccups as a side-effect. It should be emphasized that clinicians using aripiprazole for the treatment of adolescent patients should be mindful of this rare and fairly unknown phenomenon, especially in the early stages of drug initiation and while increasing the dosage.
\end{abstract}

Keywords: Aripiprazole, dopamine, hiccup, serotonin

\section{INTRODUCTION}

Hiccups are caused by sudden inspiration and then closure of the glottis right after repetitive spasmodic contractions of the diaphragm and the inspiratory muscles. It is often difficult to determine the etiology of hiccups due to the wide variety of clinical conditions, such as gastric distension, gastroesophageal reflux, or infections that may cause them (1). Studies have shown that drugs increasing the dopamine level may cause hiccups, whereas antidopaminergic drugs are used in the treatment of this condition $(2,3)$. But contrary to these findings, some researchers have reported hiccups to be caused by antidopaminergic drugs as well as by Parkinson's disease itself (4).

In the literature, there is one case report of clozapine-induced hiccups, while olanzapine and sertraline were used in the treatment of hiccups in two other cases (5-7). Considering the pharmacodynamic profiles of olanzapine, sertraline, and clozapine, it is safe to say that serotonin plays a role in the development of hiccups as well. With its partial dopamine agonistantagonist effect, aripiprazole has a distinct place among all other antipsychotics. It has the highest affinity for 5-HT2B, D2, and D3 dopamine receptors, significant affinity for 5-HT1A, 5-HT2A, 5-HT7, a1 A adrenergic, and $\mathrm{H} 1$ histamine receptors, and the least affinity for 5-HT1D, 5-HT2C, $\alpha 1 \mathrm{~B}, \alpha 2 \mathrm{~A}, \alpha 2 \mathrm{~B}, \alpha 2 \mathrm{C}, \beta 1$ and $\beta 2$ adrenergic, and $\mathrm{H} 3$ histamine receptors (8).

Hiccups are thought to be the results of complex interactions among the brain stem, respiratory areas in the brain, phrenic nerve nuclei, the reticular formation, and the hypothalamus; however, most of the pathophysiological mechanisms underlying hiccups are

How to cite this article: Besenek M. Hiccups associated with switching from quetiapine to aripiprazole in an adolescent patient. Dusunen Adam The Journal of Psychiatry and Neurological Sciences 2020;33:214-217.

Correspondence: Mert Besenek, Recep Tayyip Erdogan University Research and Training Hospital, Child and Adolescent Psychiatry Clinic, 53020 , Rize - Turkey

E-mail: mbesenek1989@gmail.com

Received: December 08, 2019; Revised: January 03, 2020; Accepted: January 13, 2020 
still unknown. A review of the literature yielded a total of fifteen studies regarding the possible involvement of aripiprazole in the etiology of hiccups; only three cases were in adolescence (9-11). Reports regarding this issue in adolescent patients are still scarce. Accordingly, we aim to contribute to the understanding of this phenomenon by presenting the case of an adolescent who had panic-like symptoms and an onset of hiccups after switching from quetiapine to aripiprazole.

\section{CASE}

Our patient, a 17-year-old male, was admitted to our outpatient unit by his parents. Psychiatric anamnesis revealed that he had been a successful student at school, but his grades and school attendance had decreased dramatically over the last several months. He had been feeling irritable for a period of time, occasionally having temper bursts during which he harmed himself by punching against the walls and windows. He could not fall asleep most nights because he did not feel tired, and although he would only sleep for 2-3 hours each night, he still did not feel the need to sleep during the day. He was feeling anxious from time to time and cried for no specific reason. He thought that his self-confidence was relatively low, but he was still highly involved in self-care routines and spending money on unnecessary things. $\mathrm{He}$ had been saving up his pocket money for some time and then spent it all in the course of one hour or so. He also stated that, even though he used to drink alcohol occasionally in the past, his drinking frequency had increased over the last 2 weeks. The patient had no previous psychiatry referrals, but he reported several time periods (could not remember the exact number or the time span) in which he felt like he was "invincible," skipped school, involved himself in physical altercations, slept less, spoke faster, and harmed himself. The patient's psychiatric evaluation showed mild acceleration of speech and thoughts, tangentialism in his thought structure, and irritable and depressive mood. The interview with his parents was in line with his complaints. He did not have a family history of any psychiatric illnesses. He scored 19 on the Young Mania Rating Scale (YMRS) (12) and 40 on the Child Depression Rating Scale (CDRS) (13), and he was diagnosed with bipolar I disorder, depressive episode with mixed features according to DSM-5 (14). The patient was initiated on quetiapine $50 \mathrm{mg} /$ day, gradually increased to $200 \mathrm{mg} /$ day. After one month of treatment with quetiapine 200 $\mathrm{mg} /$ day; he reported that he still could not sleep, felt more irritable and powerful, and started to think about ways to kill himself. He scored 23 on YMRS and 47 on CDRS; hence, gradual discontinuation of quetiapine and initiation of aripiprazole was planned. The patient's quetiapine was decreased to $100 \mathrm{mg}$ /day and aripiprazole $2.5 \mathrm{mg} /$ day was initiated.

On the next day, after being administered his daily medication of aripiprazole $2.5 \mathrm{mg}$, the patient was referred to the emergency unit with complaints of hiccups. His anamnesis revealed that he had experienced acceleration of heart rate, shortness of breath, sweating, and tremors on the first day of aripiprazole administration. On the second day of his treatment, his previously mentioned panic-like symptoms ceased but hiccups started. At the time of referral, he had been suffering hiccups for over 22 hours. His physical examination was normal, blood tests were in the normal range, and all of the potential physical and environmental causes of hiccups (such as head trauma, gastroesophageal reflux, infections) were ruled out. The Naranjo Scale was used to determine the probability score for an adverse reaction in the form of hiccups related to aripiprazole, yielding a score of 7 (probable) (15). The patient was not administered any specific medication for hiccups; aripiprazole was discontinued and hiccups gradually stopped. As his hiccups were attributed to the aripiprazole treatment, he was started on risperidone, and the dosage was gradually increased to $4 \mathrm{mg}$ /day while his remaining quetiapine was discontinued. At the last psychiatric evaluation, his mood symptoms were under control, he was euthymic with no suicidal thoughts and he did not experience any hiccups with risperidone.

\section{DISCUSSION}

The etiopathogenesis underlying the incidence of hiccups during aripiprazole treatment still remains fairly unclear. Numerous neurotransmitters, mainly dopamine, serotonin, and GABA, have been reported to play major roles in the pathophysiologic processes leading to hiccups (16). Especially case reports of successful treatment of hiccups with dopaminergic, anti-dopaminergic, and anti-serotonergic medications prove an involvement of these neurotransmitters $(2-4,6)$. Aripiprazole is a fairly new and unique secondgeneration antipsychotic with a distinct pharmacodynamic profile; it has been reported to cause hiccups as a rare side effect.

At the time of writing this case report, we were able to find a total of fifteen studies implying a relationship between hiccups and aripiprazole: Thirteen of them were case reports $(9,10,16-26)$, one was a case series involving 
four cases (11), and another one was a retrospective study with seven cases (27). Two of the case reports associated these findings with a decrease of blood sodium levels $(21,23)$. Hyponatremia was reported to take several days to manifest itself after drug administration (23). The blood electrolyte levels of our patient were in the normal range and a physical examination did not find any symptoms or signs of hyponatremia. All other predispositions to hiccups, such as head trauma, gastroesophageal reflux, or infections were ruled out. Taking all of these facts into account, hyponatremia is probably not the main explanation of our case's hiccups.

Our patient did not have any side effects while using Quetiapine or when we switched from aripiprazole to risperidone, but hiccups occurred after switching from quetiapine to aripiprazole. Quetiapine and risperidone are antagonists at both dopaminergic (D2) and serotonergic (5-HT2A, 5-HT1A, 5-HT1C, 5-HT1D) receptors, while aripiprazole has a partial agonistantagonist effect on those receptors (28). This may suggest that neurotransmitter alterations caused by aripiprazole (mainly involving dopamine and serotonin) are the likely cause of hiccups in our case. This finding is in line with three case reports where the patients had hiccups with aripiprazole but not with quetiapine (16) or risperidone $(19,25)$. The partial agonist-antagonist effect of aripiprazole on dopaminergic receptors may cause relatively increased or decreased dopaminergic levels compared to other antipsychotics, and since both high and low dopamine levels have been reported to be related to hiccups, it can be said that aripiprazole constitutes a higher risk for hiccups.

Our case report is distinct from others in the literature because hiccups started on the second day of medication right after the patient had experienced panic-like symptoms. This symptomatology may be the proof of serotonergic disruption building up to occurrence of hiccups during aripiprazole treatment. This finding is in line with reports suggesting improvement of persistent hiccups with olanzapine (5), sertraline $(6,29)$, and tandospirone, which is a new 5HT1A agonist (30). Although there is ample evidence regarding the involvement of serotonin the in pathophysiology of hiccups, the exact mechanism is still unknown. It has been postulated that, in addition to the potential effect of D2 antagonism resulting in dystonic contractions of the diaphragm, partial activation of serotonergic receptors caused by aripiprazole (especially partial agonist-antagonist effect on 5HT1A) may lead to increased activity of phrenic neurons at the spinal cord level (2).
Rechallenging with the same pharmacological agent is needed to determine the role of a drug in causing a specific side-effect. There are only three case reports where clinicians rechallenged with aripiprazole after the hiccups had resolved $(21,24,26)$, two of which reported that hiccups re-occurred $(21,24)$. We did not restart the aripiprazole treatment after our patient's hiccups had resolved; instead, we switched to risperidone. In this regard, our findings may be interpreted not as causal but as correlational and should be approached with caution. However, when all of the previous studies are taken into account, switching from a more potent D2 and $5 \mathrm{HT} 1 \mathrm{~A}$ antagonist to aripiprazole seems to increase the risk of hiccups as a side-effect. It should be emphasized that, when using aripiprazole for the treatment of adolescent patients, clinicians should be mindful of this rare and fairly unknown phenomenon, especially in the early stages of drug initiation and while increasing the dosage. We believe our case report will contribute to the literature in this respect.

\begin{tabular}{|c|c|c|}
\hline \multicolumn{2}{|c|}{ Contribution Categories } & \multirow{2}{*}{$\begin{array}{l}\text { Author Initials } \\
\text { M.B. }\end{array}$} \\
\hline \multirow{4}{*}{ Category 1} & Concept/Design & \\
\hline & Literature review & M.B. \\
\hline & Data analysis/Interpretation & M.B. \\
\hline & Case follow-up (if applicable) & M.B. \\
\hline \multirow{2}{*}{ Category 2} & Drafting manuscript & M.B. \\
\hline & Critical revision of manuscript & M.B. \\
\hline Category 3 & Final approval and accountability & M.B. \\
\hline \multirow{2}{*}{ Other } & Technical or material support & N/A \\
\hline & Supervision & N/A \\
\hline
\end{tabular}

Informed Consent: Parents of the participant gave their informed consent prior to their inclusion to the study.

Peer-review: Externally peer-reviewed.

Conflict of Interest: All authors declare no financial interests or potential conflicts of interest.

Financial Disclosure: This study did not receive any specific grants from funding agencies in the public, commercial, or not-for-profit sectors.

\section{REFERENCES}

1. Chang FY, Lu CL. Hiccup: mystery, nature and treatment. J Neurogastroenterol Motil 2012; 18:123-130.

2. Launois S, Bizec JL, Whitelaw WA, Cabane J, Derenne JP. Hiccup in adults: an overview. Eur Respir J 1993; 6:563-575.

3. Bagheri H, Cismondo S, Montastruc JL. Drug-induced hiccup: a review of the France pharmacologic vigilance database. Therapie 1999; 54:35-39.

4. Miyaoka H, Kamijima K. Perphenazine-induced hiccups. Pharmacopsychiatry 1999; 32:81. 
5. Alderfer BS, Arciniegas DB. Treatment of intractable hiccups with olanzapine following recent severe traumatic brain injury. J Neuropsychiatry Clin Neurosci 2006; 18:551-552.

6. Vaidya V. Sertraline in the treatment of hiccups. Psychosomatics 2000; 41:353-355.

7. Solla P, Congia S, Secchi L, Perra E, Cannas A. Clozapineinduced persistent hiccup in a patient with Alzheimer's disease. Clin Neurol Neurosurg 2006; 108:615-616.

8. Shapiro DA, Renock S, Arrington E, Chiodo LA, Liu LX, Sibley DR, et al. Aripiprazole, a novel atypical antipsychotic drug with a unique and robust pharmacology. Neuropsychopharmacology 2003; 28:1400-1411.

9. Kutuk MO, Guler G, Tufan AE, Kutuk O. Hiccup due to aripiprazole plus methylphenidate treatment in an adolescent with attention deficit and hyperactivity disorder and conduct disorder: a case report. Clin Psychopharmacol Neurosci 2017; 15:410-412.

10. Benyakorn S. Aripiprazole-induced persistent hiccups: a case report. J Med Assoc Thai 2017; 100:212.

11. De Filippis S, Ranieri V, Cuomo I, Zingaretti P, Kotzalidis GD, Telesforo CL, et al. Hiccup with aripiprazole plus benzodiazepines resolving with pregabalin and/or benzodiazepine switch/ discontinuation: four case reports. J Clin Psychopharmacol 2015; 35:195-197.

12. Young RC, Biggs JT, Ziegler VE, Meyer DA. A rating scale for mania: reliability, validity and sensitivity. Br J Psychiatry 1978; 133:429-435.

13. Mayes TL, Bernstein IH, Haley CL, Kennard BD, Emslie GJ. Psychometric properties of the Children's Depression Rating Scale-Revised in adolescents. J Child Adolesc Psychopharmacol 2010; 20:513-516.

14. American Psychiatric Association. Diagnostic and Statistical Manual of Mental Disorders, Fifth ed. (DSM-5). Washington DC: American Psychiatric Publishing, 2013.

15. Naranjo CA, Busto U, Sellers EM, Sandor P, Ruiz I, Roberts EA, et al. A method for estimating the probability of adverse drug reactions. Clin Pharmacol Ther 1981; 30:239-245.

16. Ray P, Zia Ul Haq M, Nizamie SH. Aripiprazole-induced hiccups: a case report. Gen Hosp Psychiatry 2009; 31:382-384.

17. Serafini G, Piccinini G, Visimberga S, Cervetti A, Belvederi Murri M, Monacelli F, et al. Aripiprazole-induced persistent hiccup: a case report and review of the literature. Psychiatr Danub 2019; 31:26-31.

18. Silverman MA, Leung JG, Schak KM. Aripiprazole-associated hiccups: a case and closer look at the association between hiccups and antipsychotics. J Pharm Pract 2014; 27:587-590.

19. Yeh YW. Persistent hiccups associated with switching from risperidone to aripiprazole in a schizophrenic patient with cerebral palsy. Clin Neuropharmacol 2011; 34:135-136.

20. Mathews M, Mathews N, Menon N, Ahmad R, Papa-Molter A. Hiccups with dose titration of aripiprazole. Prim Care Companion CNS Disord 2018; 20:17102251.

21. Behere RV, Venkatasubramanian G, Naveen MN, Gangadhar BN. Aripiprazole-induced hyponatremia: a case report. J Clin Psychiatry 2007; 68:640-641.

22. Duvarci İ, Yilmaz M. Persistent hiccups after switching from zuclopenthixol to aripiprazole. Bulletin of Clinical Psychopharmacology 2013; 23:89-90.

23. Ginsberg DL. Aripiprazole-induced hyponatremia. Prim Psychiatry 2007; 14:19-20.

24. Hori H, Nakamura J. Hiccups associated with switching from olanzapine to aripiprazole in a patient with paranoid schizophrenia. Clin Neuropharmacol 2014; 37:88-89.

25. Kattura R, Shet P. Aripiprazole induced hiccups. Ment Heal Clin 2013; 3:119-21.

26. Sakalli Kani A, Öcek T, Aksoy-Poyraz C, Turan S, Duran A. Aripiprazole-induced acute hiccups: a case report. J Neuropsychiatry Clin Neurosci 2015; 27:e60.

27. Caloro M, Pucci D, Calabrò G, de Pisa E, Mancinelli I, Rosini E, et al. Development of hiccup in male patients hospitalized in a psychiatric ward: is it specifically related to the aripiprazolebenzodiazepine combination? Clin Neuropharmacol 2016; 39:67-72.

28. Correll CU. From receptor pharmacology to improved outcomes: individualising the selection, dosing, and switching of antipsychotics. Eur Psychiatry 2010; 25:S12-21.

29. Benowitz N. Antidepressants, general (noncyclic): In Olson K (editör). Poisoning and drug overdose. Sixth Eds., New York, USA: McGraw-Hill Professional, 2012,

30. Takahashi T, Murata T, Omori M, Tagaya M, Wada Y. Successful treatment of intractable hiccups with serotonin (5-HT) $1 \mathrm{~A}$ receptor agonist. J Neurol 2004; 251:486-487. 